\title{
Revealing the interior of black holes out of equilibrium in the Sachdev-Ye-Kitaev model
}

\author{
Ram Brustein* and Yoav Zigdon $^{\dagger}$ \\ Department of Physics, Ben-Gurion University, Beer-Sheva 84105, Israel
}

(Received 28 April 2018; published 24 September 2018)

\begin{abstract}
The Sachdev-Ye-Kitaev (SYK) model can be used to describe black holes (BHs) in two-dimensional nearly anti-de Sitter gravity. We show that when such BHs are perturbed by a time-dependent negativeenergy perturbation, their interior can be partially revealed. In the SYK model, a partial measurement of the state of the Majorana fermion pairs allows one to construct a matching time-dependent negative-energy perturbation of the $\mathrm{BH}$ geometry that shifts the state of the $\mathrm{BH}$ away from its equilibrium state. Kourkoulou and Maldacena showed that, if the perturbation is strong enough, the interior can be fully exposed and the $\mathrm{BH}$ disappears. Here, we show that when the perturbation is weaker than the threshold for full exposure, it effectively moves the horizon of the $\mathrm{BH}$ inwards, thus partially exposing the interior of the $\mathrm{BH}$ and leaving behind a smaller BH. The exposure is in proportion to the number of measured Majorana pairs and so also in proportion to the magnitude of the energy of the perturbation. From the boundary, the partial measurement is perceived as a burst of radiation whose strength and duration are proportional to the number of measured Majorana pairs.
\end{abstract}

DOI: $10.1103 /$ PhysRevD.98.066013

\section{INTRODUCTION}

In classical general relativity (GR), the black hole (BH) interior is an empty region with (possibly) some singular core. Quantum theory suggests otherwise [1-5]. Yet, it is unclear whether and how it is possible for an observer outside the $\mathrm{BH}$ to determine the state of the interior. The $\mathrm{BH}$ is surrounded by a horizon which, classically, prevents such an observer from obtaining any information about the interior. Quantum mechanically, BHs in equilibrium emit Hawking radiation [6]. Because the rate of emission is so slow, an external observer has to wait for a Page time [7] until the state of the BH can start to be "read." If all of the Hawking radiation is collected (a practically impossible task), the observer would be able to determine the state of the BH. From this perspective, one can view the evaporation process as gradually exposing the state of the $\mathrm{BH}$ interior.

It was argued in $[8]$ (see also $[9,10]$ ) that, when the $\mathrm{BH}$ is out of equilibrium, it can emit "supersized" Hawking radiation-radiation of much larger amplitude than the standard Hawking radiation. Then some properties of the state of the $\mathrm{BH}$ can be determined much earlier than

\footnotetext{
ramyb@bgu.ac.il

†yoavzig@post.bgu.ac.il
}

Published by the American Physical Society under the terms of the Creative Commons Attribution 4.0 International license. Further distribution of this work must maintain attribution to the author(s) and the published article's title, journal citation, and DOI. Funded by SCOAP ${ }^{3}$. the Page time by monitoring the large-amplitude coherent emission of gravitational waves. This emitted radiation is analogous to stimulated emission, in contrast to the standard Hawking radiation which is analogous to spontaneous emission.

What does it mean to observe the state of the interior? An outside observer must interpret any radiation coming out from the $\mathrm{BH}$ as emitted from a region outside the horizon. Hence, the stimulated Hawking emission must be viewed from an outside observer perspective as induced by a negative-energy perturbation that caused the horizon to recede inwards, thus exposing part of the $\mathrm{BH}$ interior to outside observations.

In this paper we consider two-dimensional (2D) nearly anti-de Sitter $\left(\mathrm{NAdS}_{2}\right)$ BHs and, using the Sachdev-YeKitaev (SYK) model, demonstrate the validity of the ideas which were just outlined by an explicit calculation.

The SYK model [11,12] describes $N$ Majorana fermions interacting all-to-all with random coupling constants whose typical strength is $J$. In the large $N$ and the zerotemperature limit the model has a reparametrization symmetry. When the temperature is finite but small, the dynamics of this reparametrization mode is determined by the Schwarzian action [12-14] (see also [15]).

This model is interesting because it is related to nearly $\mathrm{AdS}_{2}$ gravity [16-20], for which the fundamental symmetry is that of time reparametrizations in the limit of zero dilaton field (pure GR).

The Schwarzian action describes the time reparametrization on the boundary for finite but small dilaton. 
The solution of the equation of motion (EOM) derived from the Schwarzian action describes a boundary which has a finite time extent. This bounded spacetime can be described by the exterior of an $\mathrm{AdS}_{2}$ Rindler $\mathrm{BH}$, as will be shown in detail later on.

The significance of our work is not restricted to 2D gravity because four-dimensional (near-extremal) black holes can be described by the dilaton-gravity model that we have used. This is explained, e.g., in [15]: The entropy of such black holes has a leading correction proportional to the temperature, which is captured by the Schwarzian action. The near extremality is associated with the $\mathrm{AdS}_{2}$ near-horizon geometry. Nevertheless, we do expect that our results are relevant to $\mathrm{BHs}$ out of equilibrium in general.

Recently, Kourkoulou and Maldacena (KM) [21] considered a measurement of all Majorana pairs, which, from a spacetime point of view, corresponds to a time-dependent, negative-energy perturbation which reveals the entire region behind the horizon of the $\mathrm{NAdS}_{2} \mathrm{BH}$. Thus KM showed that a strong enough perturbation corresponds to a measurement of the state of the $\mathrm{BH}$ interior. From a spacetime point of view, the $\mathrm{BH}$ disappears completely as a result of the perturbation and an outside observer can therefore fully determine the state of the interior.

Here, we assume a measurement on the subsystem of a large number of Majorana pairs and show that the $\mathrm{BH}$ interior is partially revealed due to a perturbation which takes the BH out of equilibrium. The measurement is of an essential importance. If the pairs are not measured, the average value of the perturbation Hamiltonian vanishes. If the pairs are measured, this corresponds to a negativeenergy perturbation that induces an inward shift of the horizon: some part of the geometry that was previously inside the horizon is outside the horizon after the perturbation is applied. The extent of the exposure is shown to be proportional to the number of measured Majorana pairs and also to the magnitude of the energy of the perturbation. We find that from the boundary point of view, the partial measurement is interpreted as a flow of energy in the form of radiation. A boundary observer can ascribe the source of this radiation to the interior region of the $\mathrm{BH}$ that is exposed by the perturbation.

The collision of two astrophysical BHs creates, as an intermediate state, a $\mathrm{BH}$ out of equilibrium which decays to its equilibrium state by emitting (mostly) gravitational waves. Then, following the same logic as above, the interior of the created $\mathrm{BH}$ should be partially exposed to outside observations via its stimulated emission. Recently, several scenarios were discussed $[9,10,22-25]$ in which the emission from such BHs should be different than the one predicted by GR. Therefore, our ideas could perhaps be tested by measuring the gravitational waves that they emit.

The paper is organized as follows: First, we briefly review the SYK model in the low temperature regime $1 \ll \beta J \ll N$, the $\mathrm{NAdS}_{2}$ boundary and the relation to $\mathrm{BHs}$ in equilibrium. Then, we discuss BHs out of equilibrium. We show that the "mass-term" perturbation corresponding to the measurement of a large number of Majorana pairs extends the boundary trajectory of $\mathrm{NAdS}_{2}$. We calculate the amount of extension in global coordinates and then the flux of energy that flows to the boundary. The paper ends with a summary and interpretation of our results.

\section{DESCRIPTION OF BLACK HOLES IN EQUILIBRIUM WITH THE SYK MODEL}

The SYK model [11,12] describes $N$ Majorana fermions interacting via a four-body interaction

$$
H=\sum_{i<k<l<m}^{N} j_{i k l m} \psi_{i} \psi_{k} \psi_{l} \psi_{m} .
$$

Here $\psi_{i}$ is the $i$ th Majorana field and $j_{i k l m}$ are the coupling constants, which are randomly distributed with zero mean and a variance of $\frac{3 ! J^{2}}{N^{3}}$. The constant $J$ sets the interaction strength.

In the infrared limit $\beta J=\infty$ ( $\beta$ is the inverse temperature) and in a large number of Majorana fermions $1 \ll N$ the model has a reparametrization symmetry [12-14]. When $\tau \rightarrow f(\tau)$, the two-point function of the model $G\left(\tau, \tau^{\prime}\right)=\frac{1}{N} \sum_{i=1}^{N}\left\langle T \psi_{i}(\tau) \psi_{i}\left(\tau^{\prime}\right)\right\rangle$ transforms as

$$
G\left(\tau, \tau^{\prime}\right) \rightarrow\left(f^{\prime}(\tau) f^{\prime}\left(\tau^{\prime}\right)\right)^{\frac{1}{4}} G\left(f(\tau), f\left(\tau^{\prime}\right)\right) .
$$

We will consider the case of a large number of Majorana fermions and low energies: $1 \ll \beta J \ll N$. In this regime, the SYK model describes effectively a single degree of freedom $f(t)$-the reparametrization, governed by the Schwarzian action [12-14]:

$$
S=-\frac{N \alpha_{S}}{J} \int d t\{f(t), t\}
$$

where $\alpha_{S}$ is a numerical constant and

$$
\{f(t), t\}=\left(\frac{f^{\prime \prime}}{f^{\prime}}\right)^{\prime}-\frac{1}{2}\left(\frac{f^{\prime \prime}}{f^{\prime}}\right)^{2} .
$$

We now briefly review the $\mathrm{AdS}_{2}$ spacetime. Three coordinate systems that shall be used with their respective metrics are presented in Table I. The AdS length scale is denoted by $R$. The position of the horizon in Rindler coordinates is set by a dimensionless parameter $M$ : $r_{h}=R \sqrt{M}$. The relations between the different coordinate systems are

$$
z=\frac{R \cos (\rho)}{\cos (\tau)+\sin (\rho)}, \quad T=\frac{R \sin (\tau)}{\cos (\tau)+\sin (\rho)},
$$


TABLE I. Coordinate systems in $\mathrm{AdS}_{2}$ spacetime.

\begin{tabular}{lccc}
\hline \hline Coordinates & Temporal range & Spatial range & Geometry \\
\hline Global & $-\infty<\tau<\infty$ & $-\frac{\pi}{2} \leq \rho \leq \frac{\pi}{2}$ & $\frac{d s^{2}}{R^{2}}=\frac{-d \tau^{2}+d \rho^{2}}{\cos ^{2} \rho}$ \\
Poincaré-Patch & $-\infty<T<\infty$ & $0<z<\infty$ & $\frac{d s^{2}}{R^{2}}=\frac{-d T^{2}+d z^{2}}{z^{2}}$ \\
Rindler & $-\infty<t_{R}<\infty$ & $\sqrt{M}<\frac{r}{R}<\infty$ & $d s^{2}=-\frac{\left(r^{2}-R^{2} M\right)}{R^{2}} d t_{R}^{2}+\frac{R^{2}}{r^{2}-R^{2} M} d r^{2}$ \\
\hline \hline
\end{tabular}

$$
\frac{r}{R \sqrt{M}}=\frac{\cos (\tau)}{\cos (\rho)}, \quad \tanh \left(\frac{t_{R} \sqrt{M}}{R}\right)=\frac{\sin (\tau)}{\sin (\rho)} .
$$

Below, we briefly review the essentials of $\mathrm{NAdS}_{2}$ gravity [16-19] and its relation to the SYK model and BHs.

The boundary of $\mathrm{NAdS}_{2}$ deviates from the boundary of $\mathrm{AdS}_{2}$, which is at $z=0$. It was shown in [19] that the boundary of $\mathrm{NAdS}_{2}$ can be parametrized as $(T(t), z(t)=$ $\left.\epsilon R T^{\prime}(t)\right)$ where $T(t)$ is the Poincaré time coordinate and $\epsilon$ is small. The Schwarzian action describes the boundary value $T(t)$ of $\mathrm{NAdS}_{2}$ gravity,

$$
S=-\frac{1}{8 \pi G} \bar{\phi}_{r} \int d t\{T(t), t\}
$$

where $\bar{\phi}_{r}$ is a constant and $G$ is the Newton constant in 2D.

Comparing action (3) and (7), it follows that $f(t)$ should be identified with $T(t)$ and $f^{\prime}(t)$ with $z(t)$.

A solution of the EOM derived from Eq. (3) is the following:

$$
f(t)=\frac{\pi}{J^{2} \beta} \tanh \left(\frac{\pi t}{\beta}\right)
$$

The function $f(t)$ in Eq. (8) is bounded by $\pi /\left(J^{2} \beta\right)$. Since $f(t)$ is identified with $T(t)$, it follows that the extent of time

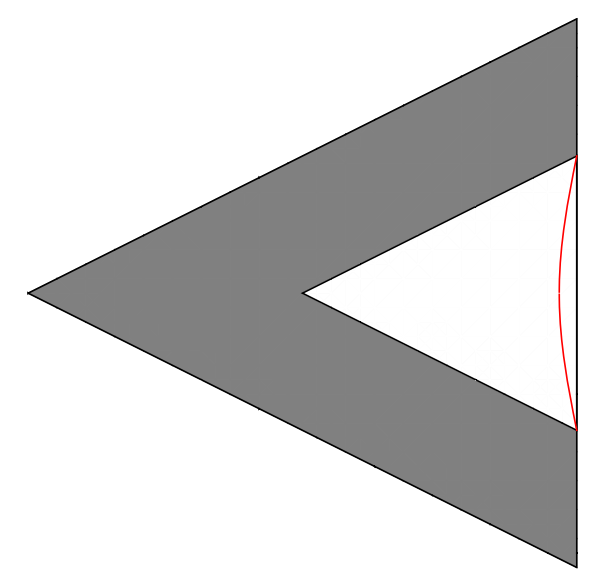

FIG. 1. The white region is accessible to the boundary observer whose world line is depicted by the (red) curve near the boundary. The shaded region describes the interior of the black hole, which is inaccessible to the observer. on the boundary of the $\mathrm{NAdS}_{2}$ is also bounded by an upper bound $T_{\text {bound }}$, i.e., $T(t) \leq T_{\text {bound }}$.

One can compactify the spacetime:

$$
\tilde{T}=\frac{R}{T_{\text {bound }}} T ; \quad \tilde{z}=\frac{R}{T_{\text {bound }}} z,
$$

therefore $\tilde{T} \leq R$. In global coordinates (5), $\tau \leq \frac{\pi}{2}$. Consider an observer on the $\mathrm{NAdS}_{2}$ boundary whose trajectory starts at $\tau=-\frac{\pi}{2}$. The accessible region for this observer in the Penrose diagram is a triangle (see Fig. 1). This region is spanned by the Rindler BH coordinates $\left(t_{R}, r\right)$, related to global coordinates as in Eq. (6). The horizon is given by the two lines $\tau= \pm \rho$.

It follows that the $\mathrm{NAdS}_{2}$ spacetime is a $\mathrm{BH}$ spacetime, whose interior corresponds to the inaccessible region for the boundary observer. This is a two-dimensional Rindler $\mathrm{BH}$ in the Poincaré-Patch.

\section{BLACK HOLES OUT OF EQUILIBRIUM}

The purpose of this section is to introduce a timedependent negative-energy perturbation to the SYK model. This perturbation depends on knowing the state of some (or all) of the Majorana pairs. We will show that when the $\mathrm{BH}$ is perturbed, a region that was previously behind the horizon becomes exposed to an outside observer.

Maldacena and Kourkoulou [21] considered the pure "spin states" $\left|B_{s}\right\rangle$, defined by

$2 i \psi_{2 k-1} \psi_{2 k}\left|B_{s}\right\rangle=s_{k}\left|B_{s}\right\rangle, \quad s_{k}= \pm 1, \quad k=1, \ldots, N / 2$

and then applied Euclidean evolution with the Hamiltonian (1) to them:

$$
\left|B_{s}(\beta)\right\rangle=e^{-\beta H / 2}\left|B_{s}\right\rangle .
$$

For $\tau=\frac{\beta}{2}+i$ and $1 \ll \beta J \ll N$, they showed that

$\left\langle B_{s}(\beta)\left|s_{k} \psi_{2 k-1}(\tau) \psi_{2 k}(\tau)\right| B_{s}(\beta)\right\rangle=-\frac{i \sqrt{\pi}}{\beta J \cosh \left(\frac{\pi t}{\beta}\right)}$.

Now, consider a subsystem of $2 j$ Majorana fermions and define the spin states only on this subsystem. We assume that $2 j<N$ and that $j$ is large. We consider the case that $j$ 
of the spins are measured, meaning that the values of $s_{k}$, $k=1, \ldots, j$ are known.

Next, construct a mass-term perturbation:

$$
V(t)=-i \epsilon J \sum_{k=1}^{j} s_{k} \psi_{2 k-1} \psi_{2 k},
$$

where $\epsilon \ll 1$. Since (13) is a sum of terms like (12), the average value of the perturbation in the state $\left|B_{s}(\beta)\right\rangle$ is

$$
\langle V(t)\rangle=-i \epsilon J \frac{-i \sqrt{\pi}}{\beta J \cosh \left(\frac{\pi t}{\beta}\right)} j=-\epsilon j \frac{\sqrt{\pi}}{\beta \cosh \left(\frac{\pi t}{\beta}\right)} .
$$

The fact that the average value of the perturbation does not vanish depends crucially on the knowledge of the state of the spins. In the state $\rho \propto I$ for which the spin values are unknown:

$$
\left\langle\psi_{2 k-1} \psi_{2 k}\right\rangle=\operatorname{tr}\left(\rho \psi_{2 k-1} \psi_{2 k}\right) \propto \sum_{\left\{s_{i}\right\}} s_{i}=0 .
$$

Therefore, $\langle V(t)\rangle=0$. Similarly, it holds for thermal states.

Equation (14) leads to a correction to the Schwarzian action. Defining $\phi$ by the derivative of the reparameterization mode, $f^{\prime}=e^{\phi}$, one obtains the action (see Appendix A):

$$
S[\phi]=\int d t\left(\frac{\dot{\phi}^{2}}{J}-J\left(e^{\phi}-\hat{\epsilon} e^{\phi / 2}\right)\right) .
$$

Here, $\hat{\epsilon} \equiv \frac{2}{\sqrt{\pi} \alpha_{S}} \epsilon \frac{j}{N}$. The EOM for $\phi$ leads to an equation expressing energy conservation:

$$
E=\frac{\dot{\phi}^{2}}{J}+J\left(e^{\phi}-\hat{\epsilon} e^{\phi / 2}\right) .
$$

The initial condition is $\dot{\phi}(t=0)=0$. The energy is a sum of a "kinetic-term" and a Liouville-type potential. The potential is depicted in Fig. 2 for three cases of varying perturbation strength: (i) unperturbed $\hat{\epsilon}=0$, (ii) small perturbation $\hat{\epsilon} \beta J \ll 1$ and (iii) large perturbation $\hat{\epsilon} \beta J>\pi$.

Using $f^{\prime}(0)=e^{\phi_{0}}$, we find that

$$
E=J\left(e^{\phi_{0}}-\hat{\epsilon} e^{\phi_{0} / 2}\right)=J \frac{\pi}{\beta J}\left(\frac{\pi}{\beta J}-\hat{\epsilon}\right) .
$$

In Appendix B we calculate in detail the perturbed solutions for $z \sim f^{\prime}=e^{\phi}$ and $T \sim f=\int d t e^{\phi}$, when $\hat{\epsilon} \beta J \ll 1$.

We can now state the main result of the paper: when the $\mathrm{BH}$ is perturbed, a region that was previously behind the horizon becomes exposed to an outside observer. The amount of exposure in global coordinates is proportional to the strength of the perturbation:

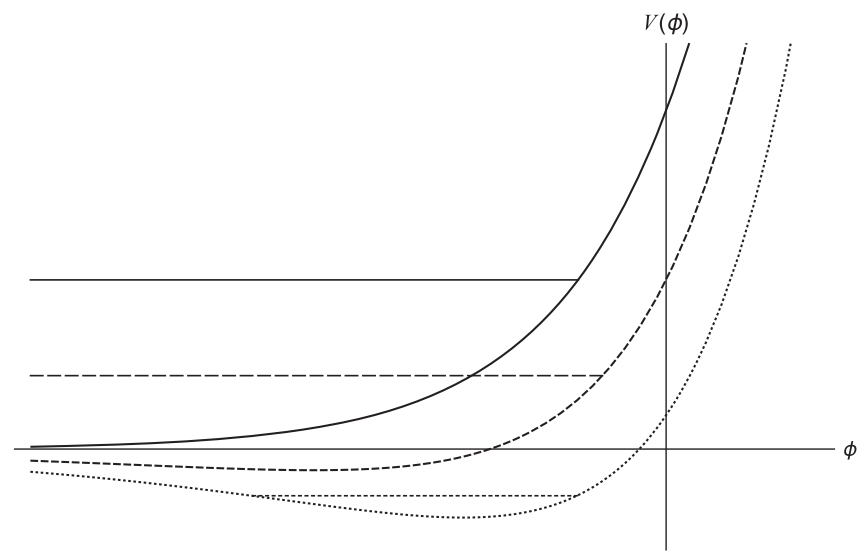

FIG. 2. Shown are the unperturbed potential (solid), the potential perturbed by a small perturbation (dashed) and the potential perturbed by a "large" perturbation (dotted). The horizontal lines show the corresponding energies.

$$
\frac{\Delta \tau}{\tau_{0}}=\frac{\pi-2}{4 \pi} \hat{\epsilon} \beta J
$$

where $\tau_{0}=\frac{\pi}{2}$. The numerical coefficient in front of the small parameter is positive: the observer will see more with respect to what she has seen in the absence of the perturbation.

The new BH horizon is given by the line $\tau=\rho+\Delta \tau$, which is a null geodesic that the observer sees at the end of her trajectory, as depicted in Fig. 3.

Next, we relate the degree of excitation to the degree of exposure. From Eq. (18),

$$
\frac{\Delta E}{E}=-\frac{\hat{\epsilon} \beta J}{\pi}
$$

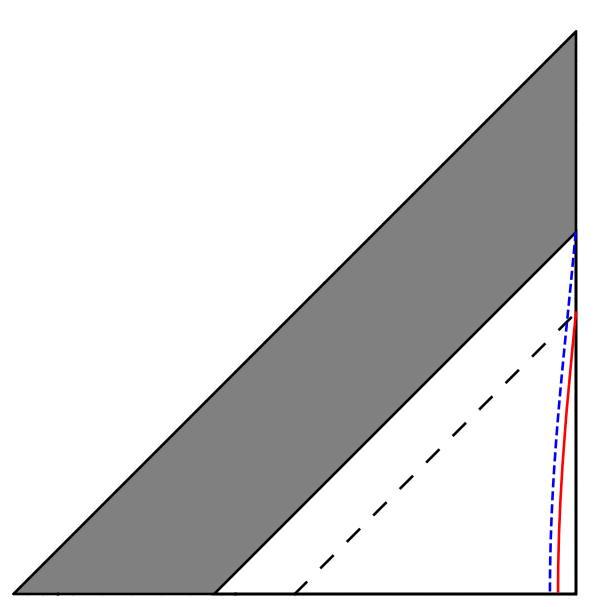

FIG. 3. The perturbation allows an observer to see a part of the interior of the unperturbed BH. A red line denotes the "old" trajectory while the blue-dashed line represents the "new" trajectory. The "old" horizon is the black dashed line while the "new" horizon is the black solid line, depicting the trajectory of a light ray leaving from the last point in the new boundary trajectory. 
It follows that

$$
\frac{\Delta \tau}{\tau_{0}}=\frac{\pi-2}{4} \frac{|\Delta E|}{E}
$$

We conclude that a measurement of $j$ spins corresponds to a small negative-energy perturbation which leads to a small region of the $\mathrm{BH}$ interior being exposed. The extent of exposure is proportional to the number of measured spins, and so also to the strength of perturbation.

We now show that a measurement of $j$ spins is interpreted at the boundary not only as the exposure of a region behind the horizon but also as a burst of radiation coming from this region. We show that if the $\mathrm{BH}$ is in equilibrium no radiation flows towards the boundary, while, in contrast, when the BH is out of equilibrium an energy flux does reach the boundary in the form of radiation.

The energy flux $F$ emitted into the bulk and arriving at the boundary is given by [19] (We work with Lorenzian signature which results an additional minus sign with respect to [19]), $F(t)=\frac{\bar{\phi}_{r}}{8 \pi G} \frac{d}{d t}\{T(t), t\}$. In SYK terms,

$$
F(t)=\frac{N \alpha_{S}}{J} \frac{d}{d t}\{f(t), t\}
$$

For a $\mathrm{BH}$ in equilibrium, $f(t) \propto \tanh (\omega t)$, which leads to $F(t)=0$ and therefore to a vanishing energy flux at the boundary.

Next, consider the measurement of $j$ Majorana spins and the "mass-term" perturbation. From Eq. (B5) in Appendix $\mathrm{B}, \omega=\frac{\pi}{2 \beta} \sqrt{1-\frac{\hat{\epsilon} \beta J}{\pi}}$ and for the case $\hat{\epsilon} \beta J<\pi$, one obtains from Eq. (B8),

$$
f^{\prime}(t)=\frac{(\pi-\hat{\epsilon} \beta J)^{2}}{(-\hat{\epsilon} \beta J+(2 \pi-\hat{\epsilon} \beta J) \cosh (\omega t))^{2}} .
$$

Substituting Eq. (23) into Eq. (22) yields the following "dimensionless flux":

$\beta^{2} F(t)=\frac{\pi^{\frac{3}{2}}}{4} \frac{\alpha_{S} N}{\beta J} \hat{\epsilon} \beta J \frac{(\pi-\hat{\epsilon} \beta J)^{\frac{3}{2}}(2 \pi-\hat{\epsilon} \beta J) \sinh (\omega t)}{((2 \pi-\hat{\epsilon} \beta J) \cosh (\omega t)-\hat{\epsilon} \beta J)^{2}}$.

Since $F(t)>0$, the energy flows to the boundary in the form of radiation. Moreover, $\beta^{2} F(t)$ is amplified by the large number $N \alpha_{S} /(\beta J)$.

For large times and $\hat{\epsilon} \beta J \ll 1$

$$
\beta^{2} F(t)=\frac{\pi^{2}}{4} \frac{\alpha_{S} N}{\beta J} \hat{\epsilon} \beta J e^{-\omega t},
$$

so the flux decays in a characteristic time $\omega^{-1}=\frac{2 \beta}{\pi \sqrt{1-\frac{\hat{\phi} \beta}{\pi}}}$ which increases as the strength of the perturbation increases.

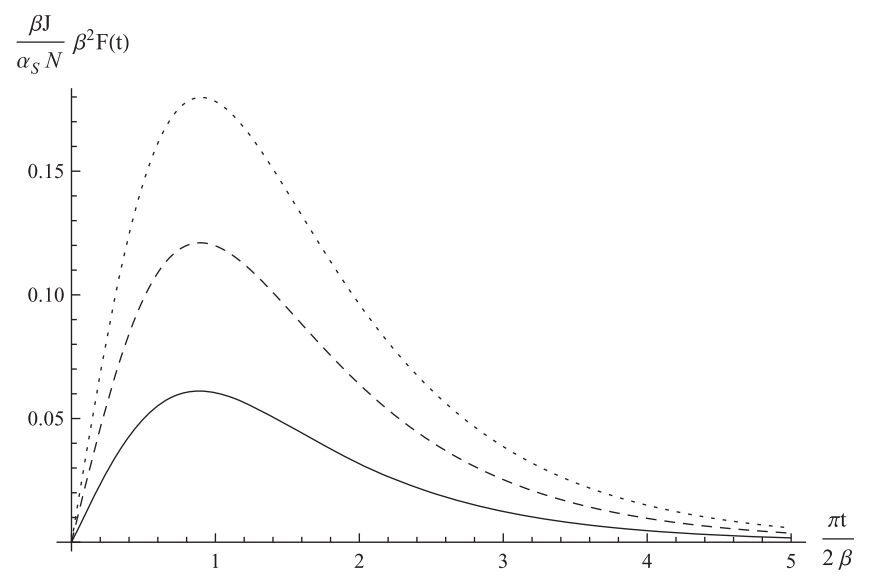

FIG. 4. The (dimensionless) energy flux into the boundary (times the factor $\beta J / N \alpha_{S}$ ) is depicted as a function of $\frac{\pi}{2 \beta} t$, for three values of perturbation strengths: $\hat{\epsilon} \beta J=0.1,0.2,0.3$ (solid, dashed and dotted, respectively).

In Fig. $4, \beta^{2} F$ (times $\frac{\beta J}{\alpha_{S} N}$ ) is plotted as a function of the dimensionless boundary time $\pi t /(2 \beta)$ for three values of $\hat{\epsilon} \beta J, \hat{\epsilon} \beta J=0.1,0.2,0.3$. The boundary observer can interpret this radiation as arriving from the region that was behind the horizon of the $\mathrm{BH}$. A phenomenon that does not occur according to classical GR.

\section{SUMMARY, INTERPRETATION AND DISCUSSION}

Using the SYK model to describe BHs in NAdS 2 gravity, we showed explicitly how a negative-energy timedependent perturbation allows an external observer to view regions of spacetime that were behind the horizon of the unperturbed $\mathrm{BH}$.

Knowing the state of $j$ spins out of $N$, we were able to construct a mass-term perturbation resulting in a change in the potential of the reparametrization mode by a magnitude $\Delta E \propto j$. The perturbation shifts the horizon of the $\mathrm{BH}$ inwards, thus exposing to an external observer a region in spacetime that used to be inside the horizon.

From the boundary, this is viewed as a burst of radiation that seems to arrive from the exposed region. This result supports the general arguments presented in [8] that external perturbations which take BHs out of equilibrium can reveal their interior via the properties of the emitted radiation while they relax to equilibrium. This idea can be perhaps verified by the detection of specific gravitational wave emission from astrophysical BH collisions, in addition to the emission predicted by GR.

\section{ACKNOWLEDGMENTS}

We would like to thank Juan Maldacena for discussions about his work and for comments on the manuscript. The research of R. B. and Y.Z. was supported by the Israel Science Foundation Grant No. 1294/16. 


\section{APPENDIX A: CORRECTED REPARAMETRIZATION MODE ACTION}

The correction to the action of the reparametrization mode $f(t)$ due to the perturbation is obtained by reparametrizing the perturbation average value as in (2),

$$
\begin{aligned}
\Delta S_{M}[\phi(t)] & =-\int d t \sqrt{\phi^{\prime}(t)}\langle V(\phi(t))\rangle \\
& =\int d t \sqrt{\phi^{\prime}(t)} \epsilon j \frac{\sqrt{\pi}}{\beta \cosh \left(\frac{\pi \phi(t)}{\beta}\right)} .
\end{aligned}
$$

The change of variables $f=\frac{\pi}{J^{2} \beta} \tanh \left(\frac{\pi \phi(t)}{\beta}\right)$ simplifies the action:

$$
\Delta S_{M}[f(t)]=\int d t \frac{1}{\sqrt{\pi}} J \epsilon j \sqrt{f^{\prime}} .
$$

The total action, together with the leading SYK term is then

$$
S=\frac{N \alpha_{S}}{2} \int d t\left(\frac{1}{J}\left(\frac{f^{\prime \prime}}{f^{\prime}}\right)^{2}+\hat{\epsilon} J \sqrt{f^{\prime}}\right)
$$

where

$$
\hat{\epsilon}=\frac{2}{\sqrt{\pi} \alpha_{S}} \epsilon \frac{j}{N} .
$$

It is useful to define $\phi(t)=\ln \left(f^{\prime}(t)\right)$ using a Lagrange multiplier $\lambda$ :

$$
S=\frac{N \alpha_{S}}{2} \int d t\left(\frac{1}{J} \dot{\phi}^{2}+\lambda\left(e^{\phi}-f^{\prime}\right)+\hat{\epsilon} J e^{\phi / 2}\right) .
$$

At $t=0$ the spin state $\left|B_{s}(\beta)\right\rangle$ in the subsystem of $j$ spins is prepared and the perturbation is turned on. For $t<0$ the solution of the EOM is $f(t)=\frac{\pi}{\beta J^{2}} \tanh \left(\frac{\pi t}{\beta}\right)$. The $\phi$ EOM at $t=0$ implies that $\lambda(t=0)=-J$. The EOM for $f$ leads to $\lambda(t)=$ const $=-J$.

Therefore the action for $\phi$ is

$$
S[\phi]=\int d t\left(\frac{\dot{\phi}^{2}}{J}-J\left(e^{\phi}-\hat{\epsilon} e^{\phi / 2}\right)\right) .
$$

\section{APPENDIX B: SOLUTION OF THE PERTURBED EQUATIONS OF MOTION}

The boundary of the unperturbed $\mathrm{NAdS}_{2} \mathrm{BH}$ ends at $\tau_{0}=\frac{\pi}{2}$. In this appendix we present the details of the calculation of the relative change in the final position of the boundary trajectory due to the perturbation, $\Delta \tau / \tau_{0}$. The perturbation corresponds to measuring $j$ Majorana pairs, for $1 \ll \beta J \ll j$. Two cases are considered:

(i) $\epsilon \hat{\beta} J>\pi$. In this case the $\mathrm{BH}$ interior is fully exposed. This case is included for completeness as it reproduces the KM result [21]. (ii) $\hat{\epsilon} \beta J \ll 1$. In this case the $\mathrm{BH}$ interior is only partially exposed.

Our calculation proceeds as follows. First, we find the solution of the energy conservation equation (17) with the initial condition $\dot{\phi}(t=0)=0$. Then, $f^{\prime} \sim e^{\phi}$ is integrated to get $T$. Finally we take the limit $t \rightarrow \infty$ and change coordinates to global coordinates.

Isolating $\dot{\phi}$ in Eq. (17) with the appropriate - sign (see Fig. 2) gives

$$
\dot{\phi}=-J e^{\phi / 2} \sqrt{\frac{E}{J} e^{-\phi}-1+\hat{\epsilon} e^{-\phi / 2}} .
$$

It is convenient to work with $x=\sqrt{\frac{|E|}{J}} e^{-\phi / 2}$ and to define

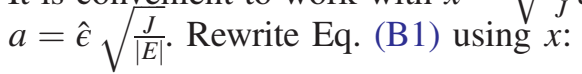

$$
2 \sqrt{\frac{J}{|E|}} \dot{x}=J \sqrt{\operatorname{sgn}(E) x^{2}-1+a x} .
$$

It follows that

$$
\frac{1}{2} \sqrt{|E| J} d t=\frac{d x}{\sqrt{\operatorname{sgn}(E) x^{2}-1+a x}} .
$$

If $E>0$, then the integration yields

$$
\frac{1}{2} \sqrt{E J} t+C_{ \pm}=\log \left(2 x+a+2 \sqrt{x^{2}-1+a x}\right) .
$$

For convenience, we define the following quantity:

$$
\omega \equiv \frac{1}{2} \sqrt{E J}=\frac{\pi}{2 \beta} \sqrt{1-\frac{\beta J \hat{\epsilon}}{\pi}} .
$$

The solution for $x$ in the case $E<0$ can be similarly found. The solution which satisfies $\dot{x}(t=0)=0$ is given by

$$
x(t)=-\frac{a}{2} \operatorname{sgn}(E)+\sqrt{1+\frac{a^{2}}{4}} \cosh (\omega t) .
$$

In order to find $e^{\phi}$, recall that (i) $a=\hat{\epsilon} \sqrt{\frac{J}{|E|}}$, (ii) $e^{-\frac{\phi}{2}}=\sqrt{\frac{J}{|E|}} x$, and (iii) $E=\frac{\pi}{\beta}\left(\frac{\pi}{\beta J}-\hat{\epsilon}\right)$. Thus,

$$
e^{-\frac{\phi}{2}}=\frac{\beta J}{2 \pi\left|\frac{\pi}{\beta J}-\hat{\epsilon}\right|}\left(-\operatorname{sgn}(E) \hat{\epsilon}+\left|\hat{\epsilon}-\frac{2 \pi}{\beta J}\right| \cosh (\omega t)\right) .
$$

The $z$ coordinate in $\mathrm{NAdS}_{2}$ is proportional to $e^{\phi}$ :

$$
z \propto e^{\phi} \propto \frac{\left(\frac{\pi}{\beta J}-\hat{\epsilon}\right)^{2}}{\left(-\operatorname{sgn}(E) \hat{\epsilon}+\left|\hat{\epsilon}-\frac{2 \pi}{\beta J}\right| \cosh (\omega t)\right)^{2}} .
$$

Below, two cases are analyzed:

(i) The case $\epsilon \hat{\beta} J>\pi$, which implies that $E<0$. For negative energies, the hyperbolic cosine in Eq. (B8) 
turns into a cosine and thus $z$ cannot approach zero for $t \rightarrow \infty$. In this case, the boundary observer never arrives at $z=0$ and so would be able to see the whole Poincaré-Patch. This reproduces the KM result.

(ii) The case $\hat{\epsilon} \beta J \ll 1$, which implies $E>0$ and thus $\omega$ is real. It follows that in this case the boundary of $\operatorname{AdS}_{2}(z \rightarrow 0)$ is met by a boundary observer for $t \rightarrow \infty$.

To determine $T$ we integrate $z$ :

$$
T(t) \sim f=\int d t f^{\prime}=\int d t e^{\phi} \propto \int d t z(t) .
$$

We keep only terms up to linear order in $\hat{\epsilon} \beta J$. In this approximation we can write $z$ as follows:

$$
\begin{aligned}
z \propto & \frac{\left(\frac{\pi}{\beta J}-\hat{\epsilon}\right)^{2}}{\left(-\hat{\epsilon}+\left(-\hat{\epsilon}+\frac{2 \pi}{\beta J}\right) \cosh (\omega t)\right)^{2}} \\
& \approx \frac{\frac{1}{4}-\frac{1}{2 \pi} \hat{\epsilon} \beta J}{\cosh ^{2}(\omega t)}\left(1+\frac{\hat{\epsilon} \beta J}{\pi} \frac{1+\cosh (\omega t)}{\cosh (\omega t)}\right) .
\end{aligned}
$$

Integrating over $t$, we find the Poincaré-Patch time:

$$
\begin{aligned}
T \propto & \text { const }+\frac{1}{4 \omega} \tanh (\omega t)-\frac{\beta J \hat{\epsilon}}{4 \pi \omega} \tanh (\omega t) \\
& +\frac{\hat{\epsilon} \beta J}{4 \pi \omega}\left(\arctan (\tanh (\omega t / 2))+\frac{1}{2} \frac{\tanh (\omega t)}{\cosh (\omega t)}\right) .
\end{aligned}
$$

Setting $T(0)=0$ requires const $=0$.
The observer meets the boundary at $z=0, t \rightarrow \infty$,

$$
T(z=0) \propto \frac{1}{4 \omega}\left(1+\hat{\epsilon} \beta J\left(\frac{1}{4}-\frac{1}{\pi}\right)\right) .
$$

To find the leading term in $\hat{\epsilon} \beta J$ we need to expand $\omega$ in (B5). Then, to leading order in $\hat{\epsilon} \beta J$,

$$
T(z=0) \propto \frac{\beta}{2 \pi}\left(1+\frac{\beta J \hat{\epsilon}}{4 \pi}(\pi-2)\right) .
$$

So,

$$
\frac{\Delta T}{T_{0}}=\frac{\hat{\epsilon} \beta J}{4 \pi}(\pi-2)
$$

It is useful to express $\Delta T / T_{0}$ in terms of the global time $\tau$. Using (5) for $\rho=\frac{\pi}{2}$,

$T\left(\rho=\frac{\pi}{2}, \tau=\frac{\pi}{2}+\Delta \tau\right)=\left.\frac{R \sin (\tau)}{1+\cos (\tau)}\right|_{\tau=\frac{\pi}{2}+\Delta \tau} \approx R+R \Delta \tau$.

Thus,

$$
\frac{\Delta \tau}{\tau_{0}}=\frac{\Delta T}{T_{0}}=\frac{\hat{\epsilon} \beta J}{4 \pi}(\pi-2)
$$

where $\tau_{0}=\frac{\pi}{2}$.
[1] A. Almheiri, D. Marolf, J. Polchinski, and J. Sully, Black holes: Complementarity or firewalls?, J. High Energy Phys. 02 (2013) 062.

[2] D. Marolf and J. Polchinski, Gauge/Gravity Duality and the Black Hole Interior, Phys. Rev. Lett. 111, 171301 (2013).

[3] S. D. Mathur, What exactly is the information paradox?, Lect. Notes Phys. 769, 3 (2009).

[4] N. Itzhaki, Is the black hole complementarity principle really necessary?, arXiv:hep-th/9607028.

[5] S. L. Braunstein, H. J. Sommers, and K. Zyczkowski, Entangled black holes as ciphers of hidden information, arXiv:0907.0739.

[6] S. W. Hawking, Particle creation by black holes, Commun. Math. Phys. 43, 199 (1975).

[7] D. N. Page, Average Entropy of a Subsystem, Phys. Rev. Lett. 71, 1291 (1993); Black hole information, arXiv:hep-th/ 9305040.

[8] R. Brustein and A. J. M. Medved, Quantum hair of black holes out of equilibrium, Phys. Rev. D 97, 044035 (2018).
[9] R. Brustein, A. J. M. Medved, and K. Yagi, When black holes collide: Probing the interior composition by the spectrum of ringdown modes and emitted gravitational waves, Phys. Rev. D 96, 064033 (2017).

[10] R. Brustein, A. J. M. Medved, and K. Yagi, Discovering the interior of black holes, Phys. Rev. D 96, 124021 (2017).

[11] S. Sachdev and J. Ye, Gapless Spin Fluid Ground State in a Random, Quantum Heisenberg Magnet, Phys. Rev. Lett. 70, 3339 (1993).

[12] A. Kitaev, A simple model of quantum holography, KITP, 2015, 2015 http://online.kitp.ucsb.edu/online/entangled15/ kitaev/, http://online.kitp.ucsb.edu/online/entangled15/ kitaev2/.

[13] A. Kitaev and S. J. Suh, The soft mode in the Sachdev-YeKitaev model and its gravity dual, J. High Energy Phys. 05 (2018) 183.

[14] J. Maldacena and D. Stanford, Remarks on the Sachdev-YeKitaev model, Phys. Rev. D 94, 106002 (2016).

[15] G. Sárosi, AdS2 holography and the SYK model, Proc. Sci. Modave2017 (2018) 001 [arXiv:1711.08482]. 
[16] A. Almheiri and J. Polchinski, Models of $\mathrm{AdS}_{2}$ backreaction and holography, J. High Energy Phys. 11 (2015) 014.

[17] K. Jensen, Chaos in $\mathrm{AdS}_{2}$ Holography, Phys. Rev. Lett. 117, 111601 (2016).

[18] J. Engelsöy, T. G. Mertens, and H. Verlinde, An investigation of $\mathrm{AdS}_{2}$ backreaction and holography, J. High Energy Phys. 07 (2016) 139.

[19] J. Maldacena, D. Stanford, and Z. Yang, Conformal symmetry and its breaking in two dimensional nearly anti-de Sitter space, Prog. Theor. Exp. Phys. 2016, $12 \mathrm{C} 104$ (2016).

[20] R. Jackiw, Lower dimensional gravity, Nucl. Phys. B252, 343 (1985); C. Teitelboim, Gravitation and Hamiltonian structure in two space-time dimensions, Phys. Lett. 126B, 41 (1983).
[21] I. Kourkoulou and J. Maldacena, Pure states in the SYK model and nearly-AdS 2 gravity, arXiv:1707.02325.

[22] V. Cardoso, E. Franzin, and P. Pani, Is the GravitationalWave Ringdown a Probe of the Event Horizon?, Phys. Rev. Lett. 116, 171101 (2016); Erratum: Is the GravitationalWave Ringdown a Probe of the Event Horizon?, Phys. Rev. Lett. 117, 089902(E) (2016).

[23] V. Cardoso, S. Hopper, C. F. B. Macedo, C. Palenzuela, and P. Pani, Gravitational-wave signatures of exotic compact objects and of quantum corrections at the horizon scale, Phys. Rev. D 94, 084031 (2016).

[24] J. Abedi, H. Dykaar, and N. Afshordi, Echoes from the abyss: The Holiday Edition!, arXiv:1701.03485.

[25] J. Abedi, H. Dykaar, and N. Afshordi, Echoes from the abyss: Tentative evidence for Planck-scale structure at black hole horizons, Phys. Rev. D 96, 082004 (2017). 